\title{
Psychological effects and risk perception after genetic counseling
}

\author{
Sunghwan Shin ${ }^{1, \oplus}$, Mi Ra Ryu ${ }^{2, \oplus}$, Won Kyung Kwon ${ }^{1 \oplus}$, Suhee Kim ${ }^{3}$, Ja-Hyun Jang ${ }^{1 \oplus}$, and Jong-Won Kim ${ }^{1} * \oplus$ \\ ${ }^{1}$ Department of Laboratory Medicine and Genetics, Samsung Medical Center, Sungkyunkwan University School of Medicine, Seoul, Korea \\ ${ }^{2}$ Department of Laboratory Medicine, Green Cross Laboratories, Yongin, Korea \\ ${ }^{3}$ Outpatient Nursing Team, Samsung Medical Center, Sungkyunkwan University School of Medicine, Seoul, Korea
}

\begin{abstract}
Purpose: Demand for genetic counseling on cancer predisposition syndrome is increasing. We evaluated the psychological effect on counselees after genetic counseling at a clinic in a single center.

Materials and Methods: We surveyed a total of 72 enrolled participants who visited a genetic counseling clinic at the Samsung Medical Center (SMC). The initial survey was conducted before the first genetic counseling session, and the second survey was conducted after the second genetic counseling session. A total of 43 participants completed both the initial and second surveys.

Results: The initial survey of 72 participants indicated higher feelings of guilt in the group with religion, higher depression and anxiety in the group with a diagnosis of self, and higher anxiety in the group on self-referral to the genetic counseling clinic. In the completed survey of 43 participants, overall decreased depression was observed after the second genetic counseling session ( $P=0.013)$. Risk perception and anxiety decreased in the group diagnosed with benign variant/variant of uncertain significance (BV/VUS, 25/3) and increased in the group diagnosed with pathogenic variant (PV, 15). Risk perception and anxiety differed between the BV/VUS and PV groups ( $P<0.001$ and $P=0.03$, respectively).

Conclusion: The genetic counseling clinic at the SMC was effective in ameliorating the depression score. Assessment of survey results revealed different depression scores, feelings of guilt and anxiety, and different effects of the genetic counseling clinic, depending on the subgroups. Understanding the needs and psychological characteristics of different groups is necessary for improving genetic counseling services.
\end{abstract}

Key words: Genetic counseling, Hereditary neoplastic syndromes.

\section{Introduction}

Along with the expansion of knowledge on a disease on a genetic basis and increasing genomic data generated by nextgeneration sequencing, the interpretation of genetic test results has been growing in complexity, and the demand for proper genetic counseling has also been increasing [1-3]. Previous studies on genetic counseling reported, increased anxiety among disease affected patients [4], decreased anxiety after genetic counseling [5], improvement in knowledge after genetic coun-

Received: 14 May 2021, Revised: 7 June 2021, Accepted: 7 June 2021, Published: 30 June 2021

${ }^{*}$ Corresponding author: Jong-Won Kim, M.D., Ph.D. (D) https://orcid.org/0000-0002-0708-9242

Department of Laboratory Medicine and Genetics, Samsung Medical Center, Sungkyunkwan University School of Medicine, 81 Irwon-ro, Gangnam-gu, Seoul 06351, Korea.

Tel: +82-2-3410-2705, Fax: +82-2-3410-2719, E-mail: kimjw@skku.edu

These authors contributed equally to this work as co-first authors.

Conflict of interest: The authors declare that they do not have any conflicts of interest.

(ac) This is an open-access article distributed under the terms of the Creative Commons Attribution Non-Commercial License (http://creativecommons.org/licenses/by-nc/4.0/) which permits unrestricted non-commercial use, distribution, and reproduction in any medium, provided the original work is properly cited.

(c) Copyright 2021 by the Korean Society of Medical Genetics and Genomics 
seling [6], more accurate risk perception status among patients with familial cancer history [7] and insignificant psychological distress after disclosure of genetic test results [8]. The most recent systematic review on outcomes of genetic counseling was conducted in 2017; however, it focused only on genetic counseling provided by non-physician genetics specialists. The study excluded genetic counseling sessions providing genetic test results, and none were from East Asian countries [5]. There is an increasing demand for culture-specific genetic counseling practice $[9,10]$ and only few studies on Koreans have been published to date $[11,12]$.

This study aimed to evaluate the psychological stress, feelings of guilt, level of anxiety, and risk perception of patients at the Samsung Medical Center genetic counseling clinic, before the first visit and after the second visit with genetic test results. The need assessment survey was also conducted to clarify patient needs for the genetic counseling clinic. This is the first study of the psychological effects of Koreans who were provided with a genetic counseling service at a medical center in Korea. We believe that a better understanding of patient response to genetic counseling sessions could lead to a better genetic counseling service targeting the unmet needs of patients.

\section{Materials and Methods}

\section{Study population and questionnaire}

We prospectively enrolled 72 adult participants who received genetic counseling at an outpatient clinic in Samsung Medical Center in July 2018. This study was approved by the Samsung Medical Center Institutional Review Board (IRB No. 2018-06041), and informed consent was obtained from all participants. All counselees were either diagnosed with cancer possibly related to cancer predisposition syndrome (CPS) or were relatives to a patient with CPS. The surveys were conducted (using questionnaires) at two different time points: before the initial genetic counseling session and after the second genetic counseling session. Genetic test was performed after the initial counseling session. Result of the genetic test was notified to the participant with explanation, during the second counseling session. Second session was usually appointed 4 weeks after the initial counseling session, unless the participant had personal circumstances to delay the appointment. A total of 43 counselees completed both the initial and second questionnaires. The questionnaire of the initial and second survey included the following information: sociodemographic data, including age, sex, occupation, religion, marital status, family size, cancer type, family history of cancer, and genetic counseling requester; previous history of mental disorder along with the magnitude of depression being assessed using the Korean version of the fourth edition of the Diagnostic and Statistical Manual of Mental Disorders, text revision (DSM-IV-TR), feelings of guilt or worthlessness, anxiety, and cancer risk perception using a numeric scale and semistructured questions; overall satisfaction and needs from genetic counseling. For the DSM-IV-TR questionnaires, the number of answers consistent with depression was counted among the 11 questions for symptoms of depression for all participants. The counted numbers were used as a depression score for each participant. Although the DSM-IV-TR is based on binary diagnostic questions (symptoms either present or absent), we used it as a semi-quantitative measure comparing participants' level of depression using 11 questions. A need assessment survey was conducted with a multiple-choice question for 72 participants in the initial survey and 43 participants in the second survey.

\section{Explanation of genetic test results}

Genetic test results are classified into three categories; pathogenic variant (PV), benign variant (BV) and variant of uncertain significance (VUS). In our study, as we explain VUS to counselees in our clinic as a non-significant result that could be regarded as similar to BV, VUS was included in the BVNVUS category for the

Table 1. Demographics and clinical characteristics of enrolled participants

\begin{tabular}{|cc|}
\hline \multicolumn{1}{|c}{ Characteristic } & Participants $(\mathrm{n}=72)$ \\
\hline Age $(\mathrm{yr})$ & $48(19-71)$ \\
\hline Sex & $14(19.4)$ \\
\hline Male & $58(80.6)$ \\
\hline Female & \\
\hline Education & $1(1.4)$ \\
\hline Elementary school & $2(2.8)$ \\
\hline Middle school & $15(20.8)$ \\
\hline High school & $46(63.9)$ \\
\hline University & $8(11.1)$ \\
Graduate school & \\
\hline Religion & $41(56.9)$ \\
Yes & $31(43.1)$ \\
\hline No & \\
\hline Have children & $46(63.9)$ \\
\hline Yes & $26(36.1)$ \\
\hline No & \\
\hline Person suspected with cancer & $32(44.4)$ \\
predisposition syndrome & $40(55.6)$ \\
\hline Patient & \\
\hline Family member & \\
\hline
\end{tabular}

Values are presented as median (range) or number (\%). 
assessment of psychological outcome after genetic counseling.

\section{Statistical analysis}

Data were analyzed using R software version 4.0.2. (R Foundation for Statistical Computing, Vienna, Austria). Numbers were expressed as mean, median, standard deviation, and interquartile range. Normality was determined using the Shapiro-Wilk test. Continuous variables were analyzed using repeated ANOVA and Wilcoxon signed-rank test for comparisons among several groups and paired Wilcoxon signed-rank test for comparison among different time points. A two-tailed $P$-value $<0.05$ was considered statistically significant.

\section{Results}

Table 1 summarizes the data on participant characteristics. Among the initially enrolled 72 participants, 43 completed the second questionnaire. The remaining 29 participants refused to or did not respond to the second questionnaire. Of the 72 participants, 32 patients were diagnosed with cancer possibly related to a cancer predisposition gene and provided an option for genetic testing targeting the suspected gene. The most common cancer was breast cancer (50\%), followed by stomach cancer (19\%). Among the 32 patients, 6 were confirmed with a PV.

The remaining 40 participants had a family member diagnosed with CPS and were provided with an option for genetic testing targeting the known pathogenic gene variant reported in the family member. The most common disease gene variant was BRCA (72\%), followed by the VHL gene (12\%). Among the 40 participants, 9 were confirmed with a PV.

While most of the enrolled participants were referred to the genetic counseling clinic by the doctor (47 participants, 65.3\%), 19 participants (26.4\%) visited a genetic counseling clinic by recommendation from a family member who was diagnosed with CPS, followed by 6 participants (8.3\%) who visited a genetic counseling clinic on self-referral.

When the assessment survey for the genetic counseling clinic was conducted for the 72 enrolled participants, 42 participants (58.3\%) answered "risk of developing cancer" and 26 participants (36.1\%) answered "cancer prevention" as needed information.

Most participants were female (80.6\%), as breast cancer patients were mostly female, and BRCA gene tests were mostly performed on females.

\section{Assessment of depression, feelings of guilt, risk perception, and anxiety}

In total, 43 participants were surveyed for depression score, feelings of guilt, risk perception, and anxiety at the initial genetic counseling session and the second genetic counseling session where genetic test results were reported. The results are presented in Table 2.

Two participants with a history of depression were excluded from the assessment of depression using DSM-IV-TR. Of the remaining 41 participants, only one met the criteria for major depression at the initial survey, and none met the criteria for major

Table 2. Assessment of depression, feelings of guilt, risk perception, and anxiety, before and after exposure to genetic test results with genetic counseling session presented by subgroups

\begin{tabular}{|c|c|c|c|c|}
\hline Characteristic & $\begin{array}{l}\text { Initial counseling } \\
\text { session }\end{array}$ & $\begin{array}{l}\text { Second counseling session } \\
\text { with genetic test results }\end{array}$ & $P$-value ${ }^{\mathrm{a}}$ & $P$-value ${ }^{b}$ \\
\hline Depression score, all $(n=41)^{c}$ & $1.27 \pm 1.91$ & $0.54 \pm 0.87$ & $0.01^{*}$ & \\
\hline Reported variant, PV (n=13) & $1.15 \pm 1.21$ & $0.54 \pm 0.78$ & 0.07 & \multirow{2}{*}{0.79} \\
\hline Reported variant, BV/VUS ( $\mathrm{n}=28)$ & $1.32 \pm 2.18$ & $0.54 \pm 0.92$ & 0.05 & \\
\hline Feelings of Guilt $(n=43)$ & $1.67 \pm 0.85$ & $1.62 \pm 0.94$ & 0.72 & \\
\hline Reported variant, PV (n=15) & $1.40 \pm 0.74$ & $1.60 \pm 0.91$ & 0.37 & \multirow{2}{*}{0.30} \\
\hline Reported variant, BV/VUS (n=28) & $1.82 \pm 0.86$ & $1.63 \pm 0.97$ & 0.38 & \\
\hline Risk perception $(n=43)$ & $1.95 \pm 0.53$ & $1.72 \pm 0.67$ & 0.06 & \\
\hline Reported variant, PV (n=15) & $2.00 \pm 0.65$ & $2.33 \pm 0.49$ & 0.12 & \multirow{2}{*}{$<0.001^{\star \star \star}$} \\
\hline Reported variant, BV/VUS (n=28) & $1.93 \pm 0.47$ & $1.39 \pm 0.50$ & $0.001^{\star \star}$ & \\
\hline Anxiety $(n=43)$ & $3.33 \pm 0.93$ & $3.16 \pm 1.31$ & 0.38 & \\
\hline Reported variant, PV (n=15) & $3.40 \pm 1.06$ & $3.80 \pm 1.26$ & 0.28 & \multirow{2}{*}{$0.03^{\star}$} \\
\hline Reported variant, BV/VUS ( $\mathrm{n}=28)$ & $3.30 \pm 0.87$ & $2.82 \pm 1.22$ & $0.04^{*}$ & \\
\hline
\end{tabular}

Values are presented as mean \pm standard deviation.

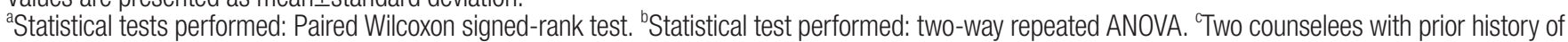
major depressive disorder were excluded from the assessment of depression score. ${ }^{\star} P<0.05,{ }^{\star \star} P<0.01,{ }^{\star \star \star} P<0.001$.

$\mathrm{PV}$, pathogenic variant; BV/VUS, benign variant/variant of uncertain significance. 
depression in the second survey. When the depression score was compared between the initial genetic counseling session and the second genetic counseling session where genetic test results were notified, a decreased mean depression score was observed $(P=0.013)$.

Analysis of guilty feelings and anxiety levels exhibited an increase or decrease in mean score after the second genetic counseling session depending on subgroups, and the result is summarized in Table 2. Lower anxiety levels were observed in the group diagnosed with BV or VUS with $P=0.04$.

While non-significant change of risk perception was observed in the group diagnosed with PV, decreased scores with a $P$-value of 0.001 were observed in the group diagnosed with BV. Risk perception differed between groups diagnosed with PV or BV, with a $P$-value of $<0.001$.

All 72 participants were surveyed at the initial visit to the genetic counseling clinic, and survey results were analyzed for depression score, feelings of guilt, risk perception, and anxiety, as shown in Table 3. When the results were compared between the group diagnosed with cancer and the group that had family members with CPS, a higher score for depression, feelings of guilt, risk perception, and anxiety were found among the group diagnosed with cancer.

The group diagnosed with cancer had higher depression score and higher anxiety level compared to family members of a cancer patient with a $P$-value of 0.02 and 0.006 , respectively. When the results were compared between the groups with and without religion, higher level of guilty feeling were observed for the group with religion $(P=0.04)$. Results were also compared between the groups with and without children. A comparison of depression score, guilty feeling, risk perception, and anxiety did not exhibit any difference. When the results were compared between the groups with different referrals, anxiety levels were significantly different according to the types of referrals $(P=0.01)$.

\section{Discussion}

To our knowledge, this is the first study on the psychological effect of genetic counseling in Korea. The perception and feelings of receiving genetic test results vary among societies $[9,10]$. We tried to assess and analyze the outcomes and effects of the genetic counseling service. Although we expected an overall decrease in depression, a decrease in feelings of guilt, better risk perception, and a decrease in anxiety after genetic counseling sessions, our survey results contrasted with our initial expectation.

When evaluated with 41 participants who answered both the initial and the second surveys without a history of depression, decreased depression scores were present in both the subgroup

Table 3. Depression score, guilty feeling, risk perception, and anxiety of counselees on initial visit to genetic counseling clinic

\begin{tabular}{|c|c|c|c|c|}
\hline Subgroup & Depression score $^{b}$ & Guilty feeling & Risk perception & Anxiety \\
\hline \multicolumn{5}{|l|}{ Initial diagnosis on } \\
\hline Patient ( $n=32)$ & $2.06 \pm 2.06$ & $2.00 \pm 0.98$ & $2.12 \pm 0.71$ & $3.81 \pm 1.06$ \\
\hline Family $(\mathrm{n}=40)$ & $1.18 \pm 1.94$ & $1.65 \pm 0.83$ & $1.98 \pm 0.48$ & $3.18 \pm 0.91$ \\
\hline$P$-value ${ }^{\mathrm{a}}$ & $0.02^{*}$ & 0.12 & 0.44 & $0.006^{\star *}$ \\
\hline \multicolumn{5}{|l|}{ Religion } \\
\hline Yes $(n=41)$ & $1.66 \pm 2.08$ & $2.00 \pm 0.97$ & $2.12 \pm 0.68$ & $3.59 \pm 1.07$ \\
\hline No $(n=31)$ & $1.45 \pm 1.99$ & $1.55 \pm 0.77$ & $1.94 \pm 0.44$ & $3.30 \pm 0.95$ \\
\hline$P$-value ${ }^{\mathrm{a}}$ & 0.6 & $0.04^{*}$ & 0.23 & 0.18 \\
\hline \multicolumn{5}{|l|}{ Have children } \\
\hline Yes $(n=46)$ & $1.76 \pm 2.26$ & $1.80 \pm 0.91$ & $2.02 \pm 0.45$ & $3.52 \pm 1.05$ \\
\hline No $(n=26)$ & $1.24 \pm 1.54$ & $1.81 \pm 0.94$ & $2.08 \pm 0.80$ & $3.36 \pm 0.99$ \\
\hline$P$-value ${ }^{\mathrm{a}}$ & 0.5 & 0.95 & 0.9 & 0.47 \\
\hline \multicolumn{5}{|l|}{ Referred to the clinic by } \\
\hline Doctor $(n=47)$ & $1.68 \pm 1.96$ & $1.87 \pm 0.90$ & $2.00 \pm 0.51$ & $3.46 \pm 1.03$ \\
\hline Self-referral $(n=6)$ & $1.00 \pm 1.55$ & $2.00 \pm 1.10$ & $2.33 \pm 0.52$ & $4.50 \pm 0.55$ \\
\hline Other family member $(n=19)$ & $1.47 \pm 2.43$ & $1.58 \pm 0.90$ & $2.05 \pm 0.78$ & $3.16 \pm 0.96$ \\
\hline$P$-value ${ }^{\mathrm{a}}$ & 0.55 & 0.33 & 0.24 & $0.01^{\star}$ \\
\hline
\end{tabular}

Values are presented as mean \pm standard deviation.

${ }^{a}$ Statistical tests performed: Wilcoxon signed-rank test. ${ }^{\mathrm{T}}$ Two counselees with prior history of major depressive disorder were excluded from the assessment of depression score. ${ }^{\star} P<0.05,{ }^{\star \star} P<0.01,{ }^{\star \star \star} P<0.001$. 
diagnosed with PV and BV/VUS. The removal of uncertainty for genetic test results could contribute to decreased depression score, which is present regardless of the reported pathogenicity of genetic variation found in the patient. In addition, psychological support from a genetic counseling clinic could have contributed to the overall decrease in depression scores. The decline of depression over genetic counseling sessions was consistent with the findings of Bjorvatn et al. [13] and Madlensky et al. [5].

Feelings of guilt were evaluated for 43 participants, as indicated in Table 2; no apparent change was observed between the initial and second genetic counseling clinics. Also when feelings of guilt were compared between the subgroup diagnosed with PV and BVNUS, no significant difference was observed between the initial and second counseling clinics. Guilty feelings of parents toward children with a disease could contribute to parental depression or anxiety $[14,15]$. Blue et al. [16] reported decreased guilt after a genetic counseling session provided for parents of children with heritable congenital heart disease. Participants diagnosed with PV might have feelings associated with concerns over heritable diseases affecting children, and they could require additional support compared to the group diagnosed with BV. Relatively smaller number of our study participants compared to Blue et al. [16], might be the cause of statistically non-significant differences between the subgroups (PV and BV/NUS).

Although risk perception exhibited an overall decrease before and after visits to the second genetic counseling clinic for the 43 participants, the trend was markedly different between the groups with $P V$ and $B V$, respectively. While risk perception exhibited a decrease in the group with BV/VUS $(P=0.001)$, no significant difference was observed in the PV group $(P=0.12)$. In addition, the results of two-way repeated-measures ANOVA for risk perception indicated a difference between the groups with $P V$ and BV $(P<0.001)$.

As risk perception is the ability to predict potential hazards related to the diagnosed genetic trait, a change in risk perception depending on the reported pathogenicity of diagnosed genetic variation is expected. More accurate risk perception depending on different risk groups after genetic counseling did align with a previous study on risk perception where Rantala et al. [7] reported participants with risk similar to general population, overestimated their risk most in the initial counseling session. However, a systematic review by Heshka et al. [17] reported no difference between carriers and noncarriers by 12 months after genetic testing. As our final survey was conducted after the second genetic counseling clinic, long-term follow-up is needed to evaluate the effectiveness of genetic counseling on patient risk perception.

In the initial survey of the 72 participants, all responses were collected before the first genetic counseling session, which was prior to notification of genetic test results. Increased anxiety and depression were noted when the suspected hereditary disease was on the participant, rather than the family member and when the participant visited the genetic counseling clinic on self-referral. People who visit the clinic on self-referral are often considered to have anxiety or depression levels well above average [18], and the result in Table 3 indicates higher anxiety and depression levels in the group on self-referral to the genetic counseling clinic. Although genetic counseling is generally not considered a stressful event, identifying individuals with higher anxiety/depression levels and allocating extended support could be of benefit [19]. Moreover, a higher level of guilty feeling was observed in the group with religion. Murakami et al. [8] reported a higher level of psychological distress associated with religion, although the result was not statistically significant.

This study has several limitations. Owing to the limited number of participants who completed the initial and the second surveys, subgroup analysis often resulted in non-significant results. In addition, our study included only a few genetic test results reported as VUS, with only three cases of VUS. Richter et al. [20] reported that the test outcome of VUS is not correctly perceived among counselees with lower education. This survey was conducted immediately before the first and after the second visit to the genetic counseling clinic, which is not appropriate for studying the long-term effects of genetic counseling. A study on the referral rate of family members for genetic testing when the counselee is confirmed with PV will also be useful for evaluating the performance of our genetic counseling clinic. In addition, hereditary diseases other than cancer were not included in this study. Rare diseases such as Huntington's disease, with unpredictable onset and poor prognosis, may result in higher psychological stress [21,22]. Assessment of psychological effects on hereditary diseases other than cancer should also be included in further studies.

In conclusion, this was the first study conducted in Korea to evaluate the psychological effect of a genetic counseling clinic on CPS. The overall effect of genetic counseling, comparing the initial and second genetic counseling sessions, was more prominent with decreased depression scores. Accurate risk perception was observed in both groups with PV and groups with BVNUS. A better understanding of the needs of counselees and the effect of the genetic counseling clinic will provide information for the proper allocation of counseling resources and the improvement 
of genetic counseling services.

\section{Acknowledgements}

This work was supported by a National Research Foundation of Korea (NRF) grant funded by the Korean government (MSIT) (No.2019R1A2C2002177).

\section{Authors' Contributions}

Conception and design: JWK. Acquisition of data: SK, MRR. Analysis and interpretation of data:SS, MRR. Drafting the article: SS. Critical revision of the article: JWK, MRR, JHJ. Final approval of the version to be published: All authors.

\section{References}

1. Yang $M, K i m$ JW. Principles of genetic counseling in the era of nextgeneration sequencing. Ann Lab Med 2018;38:291-5.

2. Prince AER, Berkman BE. Reconceptualizing harms and benefits in the genomic age. Per Med 2018;15:419-28.

3. Parens $E_{1}$ Appelbaum PS. On what we have learned and still need to learn about the psychosocial impacts of genetic testing. Hastings Cent Rep 2019;49(Suppl 1):S2-9.

4. Ballatore Z, Bracci R, Maccaroni E, Svarca L, Bianchi F, Belvederesi L, et al. Expectations and psychological issues before genetic counseling: analysis of distress determinant factors. Hered Cancer Clin Pract 2020;18:10.

5. Madlensky L, Trepanier AM, Cragun D, Lerner B, Shannon KM, Zierhut $\mathrm{H}$. A rapid systematic review of outcomes studies in genetic counseling. J Genet Couns 2017;26:361-78.

6. Braithwaite D, Emery J, Walter F, Prevost AT, Sutton S. Psychological impact of genetic counseling for familial cancer: a systematic review and meta-analysis. Fam Cancer 2006;5:61-75.

7. Rantala J, Platten $U$, Lindgren $G$, Nilsson $B$, Arver $B$, Lindblom A, et al. Risk perception after genetic counseling in patients with increased risk of cancer. Hered Cancer Clin Pract 2009;7:15.

8. Murakami Y, Okamura H, Sugano K, Yoshida T, Kazuma K, Akechi $T$, et al. Psychologic distress after disclosure of genetic test results regarding hereditary nonpolyposis colorectal carcinoma. Cancer 2004;101:395-403.

9. Pour-Jafari $H_{1}$ Pourjafari B. Lost in translation: limitations of a universal approach in genetic counseling. J Genet Couns 2010;19:5-6.

10. Wang VO. Multicultural genetic counseling: then, now, and in the 21st century. Am J Med Genet 2001;106:208-15.

11. Kang $H_{1}$ McCarthy Veach $P$, Leroy BS. Concerns of South Korean patients and family members affected with genetic conditions: a content analysis of internet website messages. J Genet Couns 2010;19:280-95.

12. Kim J, Kong SY, Han SH, Kim JW, Jeon CH, Yoo J. Genetic counseling status and perspectives based on a 2018 professional survey in Korea. Ann Lab Med 2020;40:232-7.

13. Bjorvatn C, Eide GE, Hanestad BR, Havik OE. Anxiety and depression among subjects attending genetic counseling for hereditary cancer. Patient Educ Couns 2008;71:234-43.

14. Lawoko S, Soares JJ. Distress and hopelessness among parents of children with congenital heart disease, parents of children with other diseases, and parents of healthy children. J Psychosom Res 2002;52:193-208.

15. McGill BC, Wakefield CE, Vetsch J, Lim Q, Warby M, Metcalfe A, et al. "I remember how I felt, but I don't remember the gene": families' experiences of cancer-related genetic testing in childhood. Pediatr Blood Cancer 2019;66:e27762.

16. Blue GM, Kasparian NA, Sholler GF, Kirk EP, Winlaw DS. Genetic counselling in parents of children with congenital heart disease significantly improves knowledge about causation and enhances psychosocial functioning. Int J Cardiol 2015;178:124-30.

17. Heshka JT, Palleschi $C$, Howley $H$, Wilson B, Wells PS. A systematic review of perceived risks, psychological and behavioral impacts of genetic testing. Genet Med 2008;10:19-32.

18. Brown JS, Boardman J, Elliott SA, Howay E, Morrison J. Are self-referrers just the worried well?--A cross-sectional study of self-referrers to community psycho-educational stress and self-confidence workshops. Soc Psychiatry Psychiatr Epidemiol 2005;40:396-401.

19. Nordin K, Roshanai A, Bjorvatn C, Wollf K, Mikkelsen EM, Bjelland I, et al. Is genetic counseling a stressful event? Acta Oncol 2011;50:108997.

20. Richter S, Haroun I, Graham TC, Eisen A, Kiss A, Warner E. Variants of unknown significance in BRCA testing: impact on risk perception, worry, prevention and counseling. Ann Oncol 2013;24 Suppl 8:viii6974.

21. Migliore S, Jankovic J, Squitieri F. Genetic counseling in Huntington's disease: potential new challenges on horizon? Front Neurol 2019;10:453.

22. Meiser B, Dunn S. Psychological effect of genetic testing for Huntington's disease: an update of the literature. West J Med 2001;174:33640. 